\title{
Two cases of spontaneous remission of primary hyperparathyroidism due to auto-infarction: different management and their outcomes
}

\author{
Peter Novodvorsky1,2,*, Ziad Hussein1,*, Muhammad Fahad Arshad1, Ahmed Iqbal1, \\ Malee Fernando ${ }^{3}$, Alia Munir ${ }^{1,2}$ and Sabapathy P Balasubramanian ${ }^{2,4}$ \\ 'Department of Diabetes and Endocrinology, Sheffield Teaching Hospitals NHS Foundation Trust, Sheffield, UK, \\ 2Department of Oncology and Metabolism, University of Sheffield, Sheffield, UK, ${ }^{3}$ Department of Histopathology, \\ Sheffield Teaching Hospitals NHS Foundation Trust, Sheffield, UK, and ${ }^{2}$ Department of General Surgery, Sheffield \\ Teaching Hospitals NHS Foundation Trust, Sheffield, UK \\ *(P Novodvorsky and Z Hussein contributed equally to this work)
}

\author{
Correspondence \\ should be addressed \\ to P Novodvorsky \\ Email \\ p.novodvorsky@sheffield. \\ ac.uk
}

\begin{abstract}
Summary
Spontaneous remission of primary hyperparathyroidism (PHPT) due to necrosis and haemorrhage of parathyroid adenoma, the so-called 'parathyroid auto-infarction' is a very rare, but previously described phenomenon. Patients usually undergo parathyroidectomy or remain under close clinical and biochemical surveillance. We report two cases of parathyroid autoinfarction diagnosed in the same tertiary centre; one managed surgically and the other conservatively up to the present time. Case \#1 was a 51-year old man with PHPT (adjusted (adj.) calcium: 3.11 mmol/L (reference range (RR): 2.20-2.60 mmol/L), parathyroid hormone (PTH) $26.9 \mathrm{pmol} / \mathrm{L}$ (RR: 1.6-6.9 pmol/L) and urine calcium excretion consistent with PHPT) referred for parathyroidectomy. Repeat biochemistry 4 weeks later at the surgical clinic showed normal adj. calcium $(2.43 \mathrm{mmol} / \mathrm{L})$ and reduced PTH. Serial ultrasound imaging demonstrated reduction in size of the parathyroid lesion from 33 to $17 \mathrm{~mm}$. Twenty months later, following recurrence of hypercalcaemia, he underwent neck exploration and resection of an enlarged right inferior parathyroid gland. Histology revealed increased fibrosis and haemosiderin deposits in the parathyroid lesion in keeping with auto-infarction. Case \#2 was a 54-year-old lady admitted with severe hypercalcaemia (adj. calcium: $4.58 \mathrm{mmol} / \mathrm{L}$, PTH $51.6 \mathrm{pmol} / \mathrm{L}$ (RR: 1.6-6.9 pmol/L)) and severe vitamin D deficiency. She was treated with intravenous fluids and pamidronate and 8 days later developed symptomatic hypocalcaemia $(1.88 \mathrm{mmol} / \mathrm{L})$ with dramatic decrease of PTH $(17.6 \mathrm{pmol} / \mathrm{L})$. MRI of the neck showed a $44 \mathrm{~mm}$ large cystic parathyroid lesion. To date, (18 months later), she has remained normocalcaemic.
\end{abstract}

\section{Learning points:}

- Primary hyperparathyroidism (PHPT) is characterised by excess parathyroid hormone (PTH) secretion arising mostly from one or more autonomously functioning parathyroid adenomas (up to $85 \%$ ), diffuse parathyroid hyperplasia $(<15 \%)$ and in $1-2 \%$ of cases from parathyroid carcinoma.

- PHPT and hypercalcaemia of malignancy, account for the majority of clinical presentations of hypercalcaemia.

- Spontaneous remission of PHPT due to necrosis, haemorrhage and infarction of parathyroid adenoma, the so-called 'parathyroid auto-infarction', 'auto-parathyroidectomy' or 'parathyroid apoplexy' is a very rare in clinical practice but has been previously reported in the literature.

- In most cases, patients with parathyroid auto-infarction undergo parathyroidectomy. Those who are managed conservatively need to remain under close clinical and biochemical surveillance long-term as in most cases PHPT recurs, sometimes several years after auto-infarction. 


\section{Background}

Primary hyperparathyroidism (PHPT) is characterised by excess parathyroid hormone (PTH) secretion arising mostly from one or more autonomously functioning parathyroid adenomas (up to $85 \%$ ), parathyroid hyperplasia $(<15 \%)$, and in $1-2 \%$ of cases from parathyroid carcinoma (1). PHPT with its prevalence of approximately 1:1000 $(2,3)$ is a relatively common condition and, together with hypercalcaemia of malignancy accounts for the majority of clinical presentations of hypercalcaemia. Parathyroidectomy has been considered the only definitive cure, however, watchful waiting with monitoring for the presence of end-organ damage is also warranted in selected cases (4). Over last decades, several cases of spontaneous remission of PHPT have been reported and attributed to spontaneous necrosis, haemorrhage and infarction of parathyroid adenoma and this phenomenon has been referred to as 'parathyroid auto-infarction', 'auto-parathyroidectomy' or 'parathyroid apoplexy' $(5,6,7,8,9)$.

In this report we present two cases of spontaneous resolution of PHPT due to parathyroid auto-infarction, one of which was managed surgically and the other one conservatively. We discuss their outcomes, prognosis and the strategy for long-term management of patients with this presentation.

\section{Case \#1, case presentation}

A 51-year-old man was referred to an endocrinologist by his general practitioner for further evaluation and management of suspected PHPT. He had a background of obesity, type 2 diabetes and ischaemic heart disease. His regular medications included metformin, aspirin, atorvastatin and glyceryl trinitrate spray when required. Three months prior to the referral he presented to his GP with pains and paraesthesia affecting left hand leading to a diagnosis of left carpal tunnel syndrome. There was no history of nephrolithiasis or bone fractures.

\section{Case \#1, investigation}

In the clinic, his blood pressure was $145 / 85 \mathrm{mmHg}$, height $185 \mathrm{~cm}$, weight $144 \mathrm{~kg}$ with a BMI of $43.0 \mathrm{~kg} /$ $\mathrm{m}^{2}$. His biochemistry at the time of referral showed adjusted (adj.) serum calcium $\left(\mathrm{Ca}^{2+}\right)$ of $3.11 \mathrm{mmol} / \mathrm{L}$ (reference range (RR): $2.20-2.60 \mathrm{mmol} / \mathrm{L}$ ), parathyroid hormone (PTH) 26.9 pmol/L (RR: 1.6-6.9 pmol/L) (Fig. 1), phosphate $0.49 \mathrm{mmol} / \mathrm{L}$ (RR: $0.80-1.50 \mathrm{mmol} / \mathrm{L}$ ), alkaline phosphatase 95 IU/L (RR: 30-130 IU/L), vitamin D levels of $32.9 \mathrm{nmol} / \mathrm{L}$ and the $24-\mathrm{h}$ urine calcium excretion

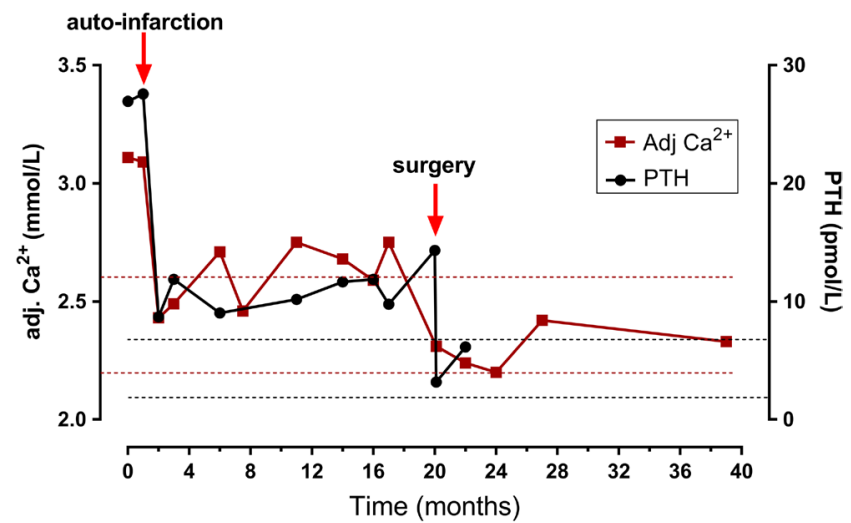

Figure 1

Case \#1. Graph showing timeline of adjusted serum $\mathrm{Ca}^{2+}$ and PTH levels with annotated times of parathyroid auto-infarction and parathyroidectomy (red arrows). Reference range for adjusted serum $\mathrm{Ca}^{2+}$ (2.20-2.60 $\mathrm{mmol} / \mathrm{L})$ is annotated with red dotted lines and reference range for PTH (1.6-6.9 pmol/L) is annotated with black dotted lines.

$18.2 \mathrm{mmol}$ (RR: $2.5-7.5 \mathrm{mmol}$ ) with urine volume of $2280 \mathrm{~mL}$. The ultrasound (US) of the neck detected a hypoechoic lobulated mass measuring $33 \times 19 \times 23 \mathrm{~mm}$ in keeping with an enlarged right inferior parathyroid gland (Fig. 2A and B). The dual scintigraphy with 99mTc sestamibi and ${ }^{99} \mathrm{Tc}$ pertechnetate showed a subtle unmatched area in the region of the left inferior posterior thyroid lobe which was suggestive of a left inferior parathyroid adenoma, however.

\section{Case \#1, treatment}

He was subsequently referred for consideration of parathyroidectomy. Four weeks later, at his pre-operative assessment, his adj. $\mathrm{Ca}^{2+}$ levels reduced spontaneously to $2.43 \mathrm{mmol} / \mathrm{L}$ and $\mathrm{PTH}$ reduced to $8.7 \mathrm{pmol} / \mathrm{L}$ (Fig. 1). A repeated US scan showed a dramatic reduction of the lesion size to $17 \times 5 \times 10 \mathrm{~mm}$ (Fig. $2 \mathrm{C}$ and D). A US-guided aspiration followed and PTH in the aspirate was found to be $84.8 \mathrm{pmol} / \mathrm{L}$ confirming parathyroid origin of this lesion. At the same time, his diabetes was noted to be poorly controlled and required initiation of insulin therapy. Given these circumstances, he was initially managed conservatively with regular serum $\mathrm{Ca}^{2+}$ and PTH monitoring (Fig. 1). Seventeen months later, his adj. $\mathrm{Ca}^{2+}$ was noted to be elevated again at $2.75 \mathrm{mmol} / \mathrm{L}, \mathrm{PTH}$ at $9.8 \mathrm{pmol} / \mathrm{L}$ and with his diabetes management now optimised, a decision was made to proceed with surgery. Twenty months after the suspected parathyroid autoinfarction, he had an uneventful bilateral neck exploration (due to discordant imaging studies as detailed above) and an enlarged right inferior parathyroid gland was identified 
A

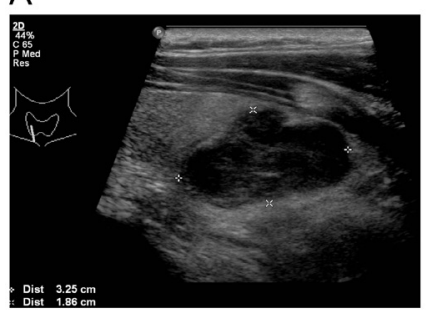

B

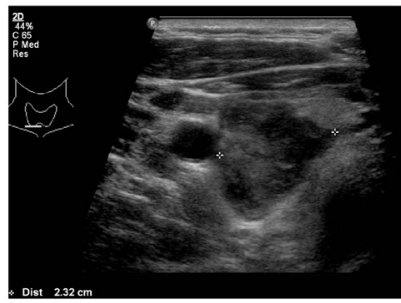

C
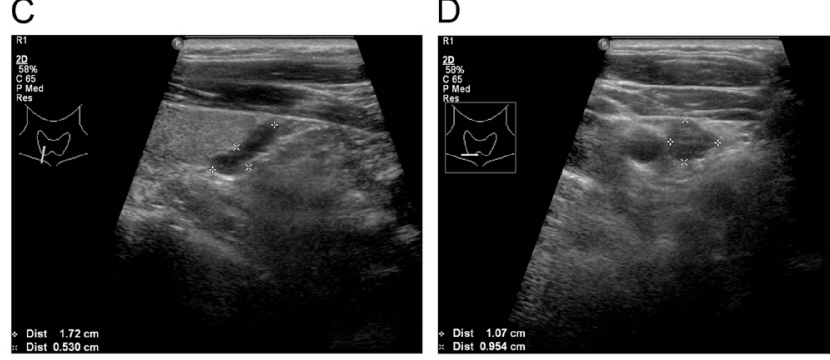

E

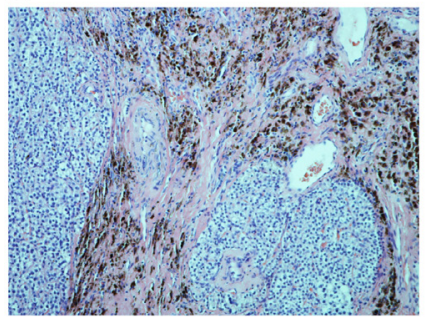

Figure 2

Case \#1. (A and B) US neck scan prior to parathyroid adenoma autoinfarction (A. long axis, B. short axis). (C and D) US neck scan several weeks after the parathyroid adenoma auto-infarction (C. long axis, D. short axis). (A, B, C and D): Parathyroid adenoma marked by white cross marks, size in left bottom corners). (E) Histopathological specimen of infarcted parathyroid adenoma (H\&E, 100x). Haemosiderin deposits and fibrosis can be observed.

and excised. The histopathological report noted increased fibrosis and haemosiderin deposits in the parathyroid gland in keeping with the previously suspected parathyroid haemorrhage and auto-infarction (Fig. 2E).

\section{Case \#1, outcome and follow-up}

On day 1 after surgery adj. serum $\mathrm{Ca}^{2+}$ was recorded at $2.31 \mathrm{mmol} / \mathrm{L}$ and PTH levels normalised as well (3.2 pmol/L). At the follow-up appointment 1.5 months after surgery his adj. $\mathrm{Ca}^{2+}$ was $2.24 \mathrm{mmol} / \mathrm{L}, \mathrm{PTH}$ was $6.2 \mathrm{pmol} / \mathrm{L}$, phosphate was $0.65 \mathrm{mmol} / \mathrm{L}$, alkaline phosphatase was $92 \mathrm{IU} / \mathrm{L}$ and vitamin $\mathrm{D}$ was $36.3 \mathrm{nmol} / \mathrm{L}$ and he was continued on calcium and vitamin D supplementation (Adcal D3 ${ }^{\circledR} 1$ tablet BD). Bone densitometry (dual-energy X-ray absorptiometry - DXA) done 2.5 months after parathyridectomy gave T-scores of +1.4 in lumbar spine, +0.4 in total hip and -2.5 in distal
$1 / 3$ of the forearm, respectively. Recommendations to continue with $\mathrm{Ca}^{2+}$ and vitamin $\mathrm{D}$ supplements were made and he could be discharged to primary care 6 months after the surgery. The serum adj. $\mathrm{Ca}^{2+}$ levels continued to be normal 6.5 months after surgery at $2.42 \mathrm{mmol} / \mathrm{L}$ (shown as month 27 in Fig. 1) and 18.5 months after surgery $2.33 \mathrm{mmol} / \mathrm{L}$ (approx. month 39 in Fig. 1).

\section{Case \#2, case presentation}

A 54-year-old lady of Afro-Carribean/mixed ethnic origin presented to Acute Medical Admissions Unit with a 3-month history of lethargy, weight loss and reduced oral intake. She didn't report any significant past medical history and wasn't taking any regular medications. There was no family history of any endocrine condition.

\section{Case \#2, investigation}

On admission, her BP was $127 / 72 \mathrm{mmHg}$, height was $172 \mathrm{~cm}$, weight was $75.2 \mathrm{~kg}$, BMI was $25.4 \mathrm{~kg} / \mathrm{m}^{2}$ and her biochemistry showed adj. serum $\mathrm{Ca}^{2+}$ of $4.58 \mathrm{mmol} / \mathrm{L}$ (RR: $2.20-2.60 \mathrm{mmol} / \mathrm{L})$, PTH at $51.6 \mathrm{pmol} / \mathrm{L}$ (RR: $1.6-$ $6.9 \mathrm{pmol} / \mathrm{L}$ ) (Fig. 3), phosphate at $0.22 \mathrm{mmol} / \mathrm{L}$ (RR: $0.80-1.50 \mathrm{mmol} / \mathrm{L})$, alkaline phosphatase at $167 \mathrm{IU} / \mathrm{L}$ (RR: 30-130IU/L), borderline elevated serum creatinine at $83 \mu \mathrm{mol} / \mathrm{L}$ (RR: $44-80 \mu \mathrm{mol} / \mathrm{L}$ ) and non-detectable vitamin D levels $(<12.5 \mathrm{nmol} / \mathrm{L})$ indicating the presence of PHPT with severe hypercalcaemia, hypophosphataemia and severe vitamin D deficiency. A computed tomography (CT) scan of thorax, abdomen and pelvis and the US scan of the neck were done within $48 \mathrm{~h}$ of her admission. The CT scan showed a $21 \mathrm{~mm}$ large right para-tracheal lesion with low density and of cystic nature (Fig. 4A). In addition, lucent bone lesions in T5 and T12 vertebrae, left ilium and left acetabulum were reported and subsequent assessment with magnetic resonance imaging (MRI) suggested these represented brown tumours secondary to PHPT. The US scan of the neck detected the presence of a heterogeneous lesion posteriorly and inferiorly to the right lobe of the thyroid with the largest dimension of $22 \mathrm{~mm}$ (Fig. 4B) and with no vascular flow within it. This lesion was identified as a right inferior parathyroid adenoma. US investigation of renal tract did not show any nephrolithiasis or nephrocalcinosis.

\section{Case \#2, treatment}

She was treated with intravenous (IV) normal saline infusion, $90 \mathrm{mg}$ of IV pamidronate and was commenced 


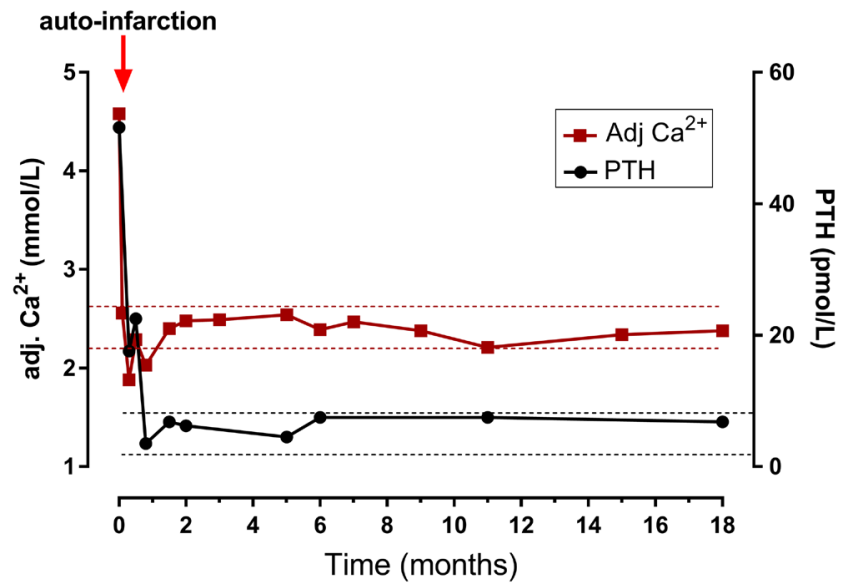

Figure 3

Case \#2. Graph showing timeline of adjusted serum $\mathrm{Ca}^{2+}$ and PTH levels. Presumed time of parathyroid auto-infarction is annotated with a red arrow. Reference range for adjusted serum $\mathrm{Ca}^{2+}(2.20-2.60 \mathrm{mmol} / \mathrm{L})$ is annotated with red dotted lines and reference range for PTH (1.6$6.9 \mathrm{pmol} / \mathrm{L}$ ) is annotated with black dotted lines.

on high-dose vitamin D supplementation (cholecalciferol 40000 units once weekly for 7 weeks). Following the initial treatment, adj. $\mathrm{Ca}^{2+}$ levels dropped significantly and 8 days after admission, she developed symptomatic hypocalcaemia (nadir adj. $\mathrm{Ca}^{2+}$ levels: $1.88 \mathrm{mmol} / \mathrm{L}$ with $\mathrm{PTH}$ at $17.6 \mathrm{pmol} / \mathrm{L}$ on day 10 post admission) which necessitated IV calcium administration (Fig. 3). A subsequent MRI neck with contrast identified a $44 \mathrm{~mm}$ large, apparently cystic lesion lying on the deep aspect of the right thyroid lobe; the medial aspect blending with tracheal wall pushing the trachea but with no airway compromise and its inferior aspect bulged into the apex of the right lung without any obvious infiltration (Fig. 4C and $\mathrm{D}$ ). These findings were supportive of the clinical suspicion of parathyroid auto-infarction. Her serum $\mathrm{Ca}^{2+}$ levels normalised several days later and she could be discharged home on cholecalciferol 40000 units once weekly for the total duration of 7 weeks together with Adcal D3 ${ }^{\circledR} 2$ tablets BD (i.e. 6 grams of $\mathrm{Ca}^{2+}$ carbonate and 1600 IU of cholecalciferol) daily. PTH levels normalised approximately 6 weeks after admission. She continued to be closely followed up in the endocrine outpatient clinic with initially monthly, later on with less frequent $\mathrm{Ca}^{2+}$ and PTH measurements (Fig. 3). Two months after the event, her vitamin D levels were recorded at $78 \mathrm{nmol} / \mathrm{L}$. A SPECT-CT imaging following application of both MIBI and pertechnetate done 3 months after initial admission demonstrated a soft tissue density inferiorly to right thyroid lobe with very low level of MIBI activity (likely due to its cystic nature) and no pertechnetate uptake, further confirming the aetiology of this lesion as a parathyroid
A
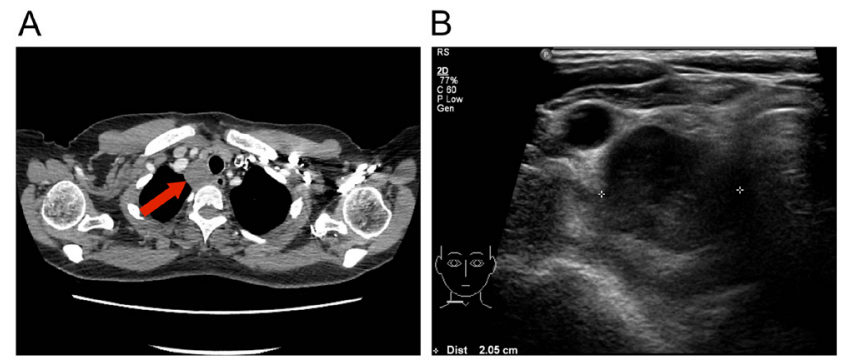

C
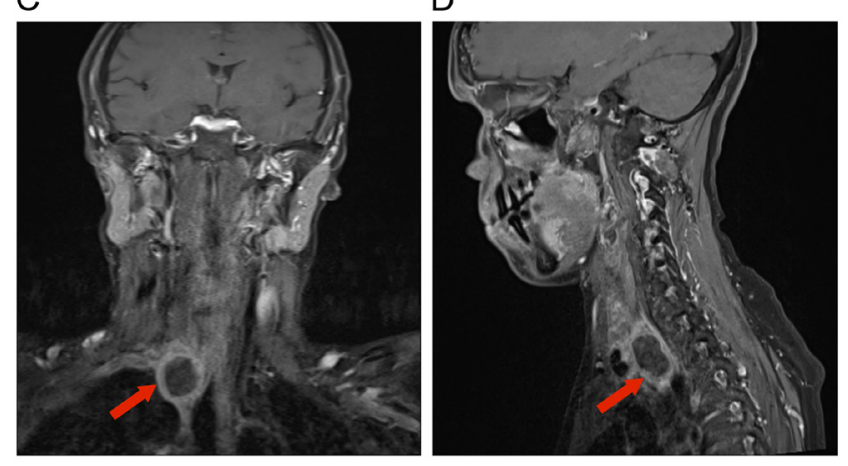

E

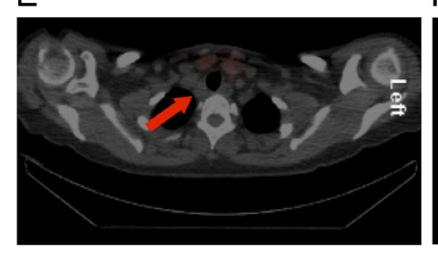

$\mathrm{F}$

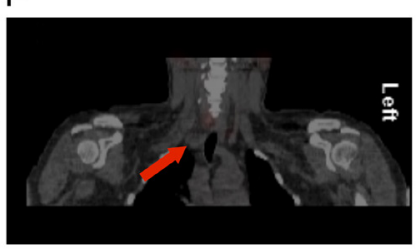

\section{Figure 4}

Case \#2. (A) CT scan of chest abdomen and pelvis showing a $21 \mathrm{~mm}$ large right para-tracheal lesion with low density and of cystic nature (red arrow). (B) US neck scan, short axis, showing a heterogeneous lesion posteriorly and inferiorly to the thyroid (annotated with white cross marks, dimension in left bottom corner). (C and D) T1-weighed MRI imaging of the neck (C. coronal view, D. sagittal view) showing a $44 \mathrm{~mm}$ large mostly cystic lesion in the lower neck (red arrows). (E and F) SPECT-CT (E. axial view, F. coronal view) following pertechnetate and MIBI administration identified the auto-infarcted parathyroid adenoma to have very low level of MIBI activity.

adenoma (Fig. 4E and F). At 6 months, the repeat MRI of the spine could not detect brown tumours anymore. At this stage, PTH levels were noted to be elevated again at $7.5 \mathrm{pmol} / \mathrm{L}$, however, with normal adj. $\mathrm{Ca}^{2+}(2.39 \mathrm{mmol} / \mathrm{L})$ and vitamin D levels $(78.9 \mathrm{nmol} / \mathrm{L})$ (Fig. 3). Similar laboratory findings were recorded at 11 months and at this stage the cystic parathyroid lesion could not be visualised on the US imaging anymore.

\section{Case \#2, outcome and follow-up}

At present, 18 months after the initial event, her adj. $\mathrm{Ca}^{2+}$ was recorded at $2.38 \mathrm{mmol} / \mathrm{L}$ and $\mathrm{PTH}$ at $6.8 \mathrm{pmol} / \mathrm{L}$ and she has remained asymptomatic. 


\section{Discussion}

Spontaneous remission of PHPT due to necrosis, haemorrhage and infarction of parathyroid adenoma, the so-called 'parathyroid auto-infarction', 'autoparathyroidectomy' or 'parathyroid apoplexy' is very rare in clinical practice but has been previously documented in the literature, the first report being published by Norris et al. in 1946 (5). Since then, more cases have been published $(6,7,8,9)$. In 1995, Nylen et al. (6) reviewed available literature and observed that presentations of parathyroid auto-infarction could be divided into two groups. In one group patients suddenly became normoor hypocalcaemic and this was sometimes linked with mild neck pain or tenderness. Necrosis, in some cases with evidence of recent or old haemorrhage, was found on histopathological examination. In the other group, patients presented more acutely with severe neck and/ or substernal pain and swelling with respiratory distress and dysphonia requiring surgical exploration. Extensive haematoma related to haemorrhage from parathyroid adenoma was found on histopathological examination (6). The authors went on to suggest a single unifying pathological process behind these presentations which begins acutely with necrosis and a degree of haemorrhage which determines the clinical presentation. In cases where haemorrhage is less severe, the process subsequently leads to fibrosis and cyst formation. The difference in the degree of haemorrhage was proposed as a defining feature for sub-classification of this disorder into three types: type 1: adenoma with necrosis without haemorrhage, type 2: adenoma with intra-capsular haemorrhage (or evidence thereof) and necrosis and type 3: adenoma with extracapsular haemorrhage (6). Parathyroid autoinfarction usually leads to acute and dramatic reduction of $\mathrm{Ca}^{2+}$ and PTH levels leading to remission of PHPT, however, in some cases, the decrease in $\mathrm{Ca}^{2+} / \mathrm{PTH}$ is much less pronounced and entirely asymptomatic (7). In most cases reported so far, surgical treatment was eventually indicated, with only a few cases reported in which conservative approach with regular clinical and biochemical follow-up was initially chosen. This might be due to contraindications for surgery or patient preference (8). In one case, PHPT recurred 7 years after the auto-infarction leading to parathyroidectomy, which was performed 8 years after the index event (8). This indicates that the clinical and biochemical surveillance has to be performed long term, perhaps life-long. The clinical observation that parathyroid auto-infarction doesn't lead to a definitive cure may be likely explained by the presence of non-ischaemic adenomatous tissue having a potential to grow resulting in recurrence of hypercalcaemia at some stage.

In our report we describe two cases of spontaneous remission of PHPT due to parathyroid auto-infarction. None of the patients could remember any neck pain or discomfort around the presumed time of the event. Both patients were initially managed conservatively. In case \#1 this was not only due to spontaneous normalisation of serum $\mathrm{Ca}^{2+}$, but also due to poorly controlled diabetes that required optimisation before surgery. Hypercalcaemia recurred 17 months later and he eventually underwent bilateral neck exploration 20 months after the index event. The histopathological findings showed fibrosis and haemosiderin deposits in keeping with parathyroid adenoma auto-infarction, type 2 as per Nylen et al.'s classification (6).

In case \#2 we have continued with conservative management up to present date, i.e. 18 months after the index event. In this case, the dramatic improvement of severe hypercalcaemia leading to symptomatic hypocalcaemia requiring IV $\mathrm{Ca}^{2+}$ administration in line with the current Society for Endocrinology Endocrine Emergency Guidance on Management of Acute Hypocalcaemia (10). These changes in serum adj. $\mathrm{Ca}^{2+}$ levels were observed after administration of pamidronate in the setting of severe vitamin D deficiency. Given this, in addition to a parathyroid auto-infarction, a plausible differential diagnosis to this clinical scenario would be the effect of bisphosphonate in the presence of severe vitamin $\mathrm{D}$ deficiency. The timing of these events (nadir $\mathrm{Ca}^{2+}$ value on day 10 after pamidronate administration) would be supportive of this. Although it is entirely possible that the above mechanism contributed to the development of symptomatic hypocalcaemia, the dramatic decrease in serum PTH levels, the cystic character of the parathyroid lesion on MRI, and inability to visualise the previously clearly visible lesion on US imaging several months later is strongly suggestive of parathyroid auto-infarction that likely occurred several days after pamidronate infusion. To our knowledge, there is only one case published in the literature linking IV administration of a bisphosphonate with subsequent parathyroid auto-infarction. This report relates to a case of a parathyroid carcinoma arising from parathyroid hyperplasia (11). Importantly, we are unaware of any plausible physiological mechanism by which bisphosphonates could cause parathyroid autoinfarction. The widespread use of bisphosphonates versus the rare incidence of parathyroid auto-infarction suggests that bisphosphonate administration in these cases was likely to be coincidental. 
In case\# 1 we had discordant results in localisation studies of the parathyroid adenoma. The US scan of the neck identified a large $\mathrm{R}$ inferior parathyroid adenoma, whereas the dual scintigraphy with ${ }^{99 m}$ Tc sestamibi (MIBI) and ${ }^{99 \mathrm{~m} T c}$ pertechnetate showed a subtle unmatched area in the region of the left inferior posterior thyroid lobe. Thus, the MIBI findings were more subtle than the findings on US imaging and as they could not be confirmed in surgery they clearly represent a false positive finding. Discordant results in various imaging modalities of the parathyroid is a well described phenomenon (12). False-positive scintigraphic findings can be caused by a variety of conditions, the most common being solitary thyroid adenoma, followed by benign and malignant lesions of the neck, breast or lung, thymic lesions or reactive lymph nodes (13). Although the sensitivity of the ${ }^{99 \mathrm{~m} T c}$ sestamibi scan is high in patients with solitary parathyroid adenomas, false-negative and -positive results of $99 \mathrm{~m} T c$ sestamibi scanning are unavoidable. There are several factors that influence scintigrafic detection of parathyroid adenomas. Apart from size (weight), ${ }^{99 \mathrm{~m} T c}$ sestamibi uptake also depends on the content of mitochondria-rich oxyphil cells, the expression of P-glycoprotein and the perfusion and functional activity of the adenoma (14). Although we are unable to provide more details on the histopathological and immunohistochemical analysis of the parathyroid adenoma specimen in case\#1, we assume that the unfavourable properties in relation to the above characteristics have contributed to the fact that, despite its size, it was not picked up on the parathyroid scintigraphy. From clinical perspective, discordant localisation studies should lead to indication of bilateral neck exploration. If at exploration no discrete abnormality can be found at the presumed site of uptake, like in was in our case\#1, this area is left alone.

In relation to the post-operative management in case\# 1 , the biochemical picture of mild hypophosphataemia at 1.5 months after surgery $(0.65 \mathrm{mmol} / \mathrm{L}$, normal RR: 0.80 $1.50 \mathrm{mmol} / \mathrm{L}$ ) and PTH levels of $6.2 \mathrm{pmol} / \mathrm{L}$ in the upper end of normal RR (1.6-6.9 pmol/L) were attributed to be secondary to ongoing vitamin D deficiency $(36.3 \mathrm{nmol} / \mathrm{L})$. The possibility of persistent mild PHPT (for example due to a second adenoma and discordant localisation studies) after bilateral neck exploration and normal adjusted $\mathrm{Ca}^{2+}$ levels was considered as very unlikely and the patient was discharged to primary care.

Lastly, in case\# 1 FNA was used to confirm the aetiology of the lesion. The high detected PTH concentration $(800 \mathrm{ng} / \mathrm{L})$ was confirmatory of its parathyroid origin. Similarly to case\#1, we considered the use of FNA, but the lesion could not be visualised on subsequent imaging; probably as a result of previous auto-infarction. It has to be noted that FNA does not form part of standard diagnostic workup in cases of suspected PHPT (4) and its indication in these circumstances is still a subject of debate. The unusual clinical behaviour of both presented cases, however, raised questions about the aetiology of the detected neck lesions and concerns were raised not to miss an alternative diagnosis (reactive lymph node, lymphoma). Both cases were discussed on the local endocrine multidisciplinary team (MDT) meetings and it was felt that the benefits of establishing the correct diagnosis outweighed the risks linked with the procedure itself including discomfort, pain or bleeding, but mainly the risk of tumour dissemination in cases of confirmed malignancy. The use of FNA under such and similar circumstances is based on our previous experience and has been reported by our group (15) as well as others $(16,17)$.

In summary, our current report adds to the already available amount of literature on cases of spontaneous PHPT remission due to parathyroid auto-infarction, provides a discussion on the possible approaches of clinical management and makes the wide readership aware of this rare, but well described phenomenon.

\section{Declaration of interest}

The authors declare that there is no conflict of interest that could be perceived as prejudicing the impartiality of the research reported.

\section{Funding}

This research did not receive any specific grant from any funding agency in the public, commercial or not-for-profit sector.

\section{Patient consent}

The authors confirm that written informed consent was obtained from both patients for publication of this article through their signatures on the consent form.

\section{Author contribution statement}

$\mathrm{P} \mathrm{N}$ did a literature search and wrote the manuscript. $\mathrm{Z} \mathrm{H}$ attended to patient $\# 2$, reviewed this case and reviewed the manuscript. M F A and A I reviewed the manuscript. M F acquired and reported the histopathological samples and reviewed the manuscript. A M is the consultant in charge for patient \#2 and reviewed the manuscript. S B was the consultant in charge for patient \#1, operated on patient \#1 and reviewed the manuscript. Case \#2 was presented by $\mathrm{Z} \mathrm{H}$ and $\mathrm{A} \mathrm{M}$ as a poster on the Society for Endocrinology BES annual meeting, 6th-11th November 2017, Harrogate, UK.

\section{Acknowledgements}

The authors would like to thank to Dr Matthew Bull and Dr Christopher Johns from the Department of Radiology, Sheffield Teaching Hospitals NHS Foundation Trust, Sheffield, UK for their help with interpretation of the scans. 


\section{References}

1 Bringhurst FR, Demay MB \& Kronenberg HM. Hormones and disorders of mineral metabolism. In Williams Textbook of Endocrinology, 13th ed, ch. 28, pp 1254-1322. Eds S Melmed, KS Pollonsky, P Reed Larsen \& HM Kronenberg. Philadelphia: Elsevier, 2016. (https://doi.org/10.1016/C2013-0-15980-6)

2 Wermers RA, Khosla S, Atkinson EJ, Achenbach SJ, Oberg AL, Grant CS \& Melton LJ 3rd. Incidence of primary hyperparathyroidism in Rochester, Minnesota, 1993-2001: an update on the changing epidemiology of the disease. Journal of Bone and Mineral Research 200621 171-177. (https://doi.org/10.1359/ JBMR.050910)

3 Boonstra CE \& Jackson CE. Serum calcium survey for hyperparathyroidism: results in 50,000 clinic patients. American Journal of Clinical Pathology 197155 523-526. (https://doi. org/10.1093/ajcp/55.5.523)

4 Bilezikian JP, Brandi ML, Eastell R, Silverberg SJ, Udelsman R, Marcocci C \& Potts JT Jr. Guidelines for the management of asymptomatic primary hyperparathyroidism: summary statement from the fourth International Workshop. Journal of Clinical Endocrinology and Metabolism 201499 3561-3569. (https://doi. org/10.1210/jc.2014-1413)

5 Norris EH. Primary hyperparathyroidism; a report of five cases that exemplify special features of this disease (infarction of a parathyroid adenoma; oxyphil adenoma). Archives of Pathology 194642 261-273.

6 Nylen E, Shah A \& Hall J. Spontaneous remission of primary hyperparathyroidism from parathyroid apoplexy. Journal of Clinical Endocrinology and Metabolism 199681 1326-1328. (https://doi. org/10.1210/jcem.81.4.8636326)

7 Kovacs KA \& Gay JD. Remission of primary hyperparathyroidism due to spontaneous infarction of a parathyroid adenoma. Case report and review of the literature. Medicine 199877 398-402. (https://doi. org/10.1097/00005792-199811000-00005)

8 Pereira FA, Brandao DF, Elias J Jr \& Paula FJ. Parathyroid adenoma apoplexy as a temporary solution of primary hyperparathyroidism: a case report. Journal of Medical Case Reports 20071 139. (https://doi. org/10.1186/1752-1947-1-139)

9 Efremidou EI, Papageorgiou MS, Pavlidou E, Manolas KJ \& Liratzopoulos N. Parathyroid apoplexy, the explanation of spontaneous remission of primary hyperparathyroidism: a case report. Cases Journal 20092 6399. (https://doi.org/10.1186/1757$1626-2-6399)$

10 Turner J Gittoes N Selby P and the Society for Endocrinology Clinical Committee. SOCIETY FOR ENDOCRINOLOGY ENDOCRINE EMERGENCY GUIDANCE: Emergency management of acute hypocalcaemia in adult patients. Endocrine Connections 5 G7-G8. (https://doi.org/10.1530/EC-16-0056)

11 de Papp AE, Kinder B, LiVolsi V, Gupta SM \& Stewart AF. Parathyroid carcinoma arising from parathyroid hyperplasia: autoinfarction following intravenous treatment with pamidronate. American Journal of Medicine 199497 399-400. (https://doi.org/10.1016/00029343(94)90312-3)

12 Mihai R, Gleeson F, Buley ID, Roskell DE \& Sadler GP. Negative imaging studies for primary hyperparathyroidism are unavoidable: correlation of sestamibi and high-resolution ultrasound scanning with histological analysis in 150 patients. World Journal of Surgery 200630 697-704. (https://doi.org/10.1007/s00268-005-0338-9)

13 Eslamy HK \& Ziessman HA. Parathyroid scintigraphy in patients with primary hyperparathyroidism: 99mTc sestamibi SPECT and SPECT/ CT. RadioGraphics 200828 1461-1476. (https://doi.org/10.1148/ rg.285075055)

14 Erbil Y, Kapran Y, Issever H, Barbaros U, Adalet I, Dizdaroglu F, Bozbora A, Ozarmagan S \& Tezelman S. The positive effect of adenoma weight and oxyphil cell content on preoperative localization with $99 \mathrm{mTc}$-sestamibi scanning for primary hyperparathyroidism. American Journal of Surgery 2008195 34-39. (https://doi.org/10.1016/j.amjsurg.2007.01.040)

15 Truran P, Stedman T, Balasubramanian S \& Harrison BJ. The utility of ultrasound guided fine needle aspiration for PTH levels in challenging cases of primary hyperparathyroidism. In British Association of Endocrine and Thyroid Surgeons Annual Scientific Conference, pp S79-S88. Reading/Henley on Thames, UK, 2015.

16 Aydin C, Polat SB, Dellal FD, Kaya C, Dogan HT, Turkolmez S, Kilic M, Ersoy R \& Cakir B. The diagnostic value of parathyroid hormone washout in primary hyperparathyroidism patients with negative or equivocal 99mTc-MIBI results. Diagnostic Cytopathology 201947 94-99. (https://doi.org/10.1002/dc.24065)

17 Abraham D, Sharma PK, Bentz J, Gault PM, Neumayer L \& McClain DA. Utility of ultrasound-guided fine-needle aspiration of parathyroid adenomas for localization before minimally invasive parathyroidectomy. Endocrine Practice 200713 333-337. (https://doi. org/10.4158/EP.13.4.333)

Received in final form 27 March 2019

Accepted 12 April 2019 